\title{
1 Oxytocin increases after affiliative interactions in male 2 Barbary macaques
}

3 Alan V. Rincon a,b, Tobias Deschnerc, Oliver Schülkee, ${ }^{\mathrm{a}, \mathrm{b}, \mathrm{d}}$, Julia Ostner ${ }^{\mathrm{a}, \mathrm{b}, \mathrm{d}}$

4 aDepartment of Behavioral Ecology, Johann-Friedrich-Blumenbach Institute for Zoology and

5 Anthropology, University of Goettingen, Goettingen, Germany

6 beibniz ScienceCampus Primate Cognition, Goettingen, Germany

7 cDepartment of Primatology, Max Planck Institute for Evolutionary Anthropology, Leipzig,

8 Germany

$9{ }^{d}$ Research Group Social Evolution in Primates, German Primate Center, Leibniz Institute for

10 Primate Research, Goettingen, Germany

11 Corresponding author: Alan V. Rincon, Department of Behavioral Ecology, University of

12 Goettingen, Kellnerweg 6, 37077 Goettingen, Germany

13 Email: avrincon1@gmail.com 


\section{Abstract}

15 Mammals living in stable social groups often mitigate the costs of group living through the

16 formation of social bonds and cooperative relationships. The neuropeptide hormone

17 oxytocin (OT) has been proposed to promote both bonding and cooperation although only

18 a limited number of studies have investigated this under natural conditions. Our aim was to

19 assess the role of OT in bonding and cooperation in male Barbary macaques (Macaca

20 sylvanus). First we tested for an effect of affiliation - grooming and triadic male-infant-male

21 interactions - with bond and non-bond partners on urinary OT levels. Secondly we aimed to

22 test whether grooming interactions (and thus increased OT levels) increase a male's

23 general propensity to cooperate in polyadic conflicts. We collected behavioral data via full-

24 day focal animal protocols on 14 adult males and measured endogenous OT levels from

25139 urine samples collected after affiliation and non-social control periods. Urinary OT

26 levels were higher after grooming with any partner. By contrast, OT levels after male-

27 infant-male interactions with any partner or with bond partners were not different from

28 controls but were higher after interactions with non-bond partners. Previous grooming did

29 not increase the likelihood of males to support others in conflicts. Collectively, our results

30 support research indicating that OT is involved in the regulation of adult social bonds,

31 including in non-reproductive contexts. However, our male-infant-male interaction results

32 go against previous studies suggesting that it is affiliation with bond rather than non-bond

33 partners that trigger the release of OT. Alternatively, OT levels may have been elevated

34 prior to male-infant-male interactions thus facilitating interaction between non-bond

35 partners. The lack of an association of grooming (and by extension increased OT levels) and

36 subsequent support speaks against an OT linked increase in the general propensity to

37 cooperate, yet further studies are needed for a more direct test including the possibility of

38 partner-specific contingent cooperation.

39 Keywords: oxytocin, social bonds, cooperation, grooming, male-infant-male interaction,

40 Barbary macaque 


\section{Introduction}

42 For mammals living in stable social groups, investing in strong social bonds can provide

43 individuals with adaptive benefits (Ostner and Schülke, 2018) such as increased

44 reproductive success (Cameron et al., 2009; Frère et al., 2010; Schülke et al., 2010; Strauss

45 and Holekamp, 2019; Weidt et al., 2008) and increased survival (Archie et al., 2014; Giles et

46 al., 2005; Silk et al., 2010). Social bonds are usually characterized by high rates of affiliative

47 interactions (Ostner and Schülke, 2018) and may promote cooperative behavior (Schülke

48 et al., 2010; Smith et al., 2011; Weidt et al., 2008; Young et al., 2014b) and buffer

49 physiological impacts of stress (Cheney and Seyfarth, 2009; Young et al., 2014a).

50 One hormone implicated in the formation and maintenance of social bonds is the highly

51 conserved neuropeptide oxytocin (OT). OT plays a role in promoting maternal behavior

52 (Finkenwirth et al., 2016; Ross and Young, 2009) and ultimately a partner specific

53 attachment between mother and offspring (Ross and Young, 2009). The oxytocinergic

54 system is thought to have been co-opted from its ancestral function of mother-offspring

55 attachment to also promote social bonds between adults (Numan and Young, 2016; Ziegler

56 and Crockford, 2017). This has been best demonstrated in the context of pair bonds where

57 OT helps regulate a social preference for a particular mating partner (French et al., 2018;

58 Ross and Young, 2009). Oxytocin may also regulate social bonds more broadly outside the

59 pair-bond and in non-reproductive contexts. In support of this, OT is released after

60 positive, non-sexual, social interactions (chimpanzees, Pan troglodytes: Crockford et al.,

61 2013; Preis et al., 2018; Wittig et al., 2014; tufted capuchins, Sapajus apella: Benítez et al.,

62 2018; dogs, Canis familiaris: Romero et al., 2014). Because OT interacts with the reward

63 system (Dölen et al., 2013; Love, 2014; Skuse and Gallagher, 2009), OT release potentially

64 stimulates a 'feel good' sensation after positive social interactions. These sensations may be

65 part of the mechanism by which social bonds are maintained (e.g., via emotional

66 bookkeeping: Schino and Aureli, 2009).

67 OT release is partner specific, at least in some studies. In chimpanzees, urinary OT levels

68 are increased after grooming with a bonded partner, but not after the same interaction

69 with a non-bonded partner (Crockford et al., 2013). Similarly, in cooperatively breeding 
marmosets (Callithrix jacchus), strongly bonded family members show synchronous

71 fluctuations in baseline urinary OT levels whereas weakly bonded partners do not,

72 suggesting that affiliation with bond partners influences OT levels more than affiliation

73 with non-bond partners (Finkenwirth et al., 2015). Other studies, however, suggest that OT

74 is released independently of partner bond strength (Preis et al., 2018; Wittig et al., 2014) or

75 that the impact of bond partner strength on OT secretion depends on the type of

76 interaction (Wittig et al., 2014).

77 In addition to social bonding, oxytocin plays a key role in promoting coordination and

78 cooperative behaviors under certain contexts. In economic games, intranasal

79 administration of OT increases cooperation when participants had prior contact but not

80 when they were anonymous (Declerck et al., 2010), and similarly in in-group but not out-

81 group conditions (De Dreu et al., 2010; Ten Velden et al., 2017). Performance on a

82 cooperative task was also improved by OT administration, suggestive of OT's role in the

83 facilitation of coordination of behavior (Arueti et al., 2013). These findings in humans are

84 paralleled in chimpanzees where urinary OT levels were elevated during coordinated

85 behaviors such as territorial border patrols (Samuni et al., 2017) and cooperative hunting

86 (Samuni et al., 2018) compared to controls. Furthermore, the highest levels of urinary OT

87 in chimpanzees occurred during inter-group encounters, which involve joint aggression

88 against out-group members (Samuni et al., 2017). Depending on context, OT appears to

89 facilitate participation in polyadic aggression through increased coordination and in-group

90 favoritism in humans and chimpanzees. The generality of these patterns beyond these taxa

91 remains to be tested.

92 We aimed to investigate the role of oxytocin in the maintenance of social bonds and

93 cooperation in male macaques. Macaque males of several species form strong, equitable

94 and stable social bonds with other males (Kalbitz et al., 2016; Young et al., 2014b) which

95 are predictive of cooperation via coalition formation (Berghänel et al., 2011; Schülke et al.,

96 2010; Young et al., 2014b). Coalitions serve to increase or maintain male dominance rank

97 (Young et al., 2014c), and increase mating success (Küster and Paul, 1992; Young et al.,

98 2013) or reproductive success (Schülke et al., 2010). In addition to male-male bonds,

99 macaques may also from strong male-female bonds (Haunhorst et al., 2016; Massen and 
100 Sterck, 2013). Males frequently support females in agonistic conflicts (Haunhorst et al.,

101 2017; Kulik et al., 2012; Small, 1990), and similarly to male-male relations, the probability

102 to support is predicted by social bond strength (Haunhorst et al., 2017; Kulik et al., 2012).

103 A behavioral pattern proposed to enhance male social bonding are triadic male-infant-male

104 interactions which are characteristic yet not exclusive to Barbary macaques (Deag, 1980;

105 Paul et al., 1996). These interactions are ritualistic in nature and involve two males sitting

106 in body contact holding an infant in between them while teeth-chattering and often

107 inspecting the infant's genitals (Deag, 1980; Deag and Crook, 1971). This behavior most

108 commonly involves newborn infants, although yearlings and two-year-olds are also

109 sometimes involved (Paul et al., 1996). Triadic male-infant-male interactions have been

110 proposed to be used as a tool to enhance male-male social bonds (Henkel et al., 2010;

111 Kalbitz et al., 2017; Paul et al., 1996) and predict coalition formation in the mating season

112 (Berghänel et al., 2011). Alternatively, though not mutually exclusive, male-infant-male

113 interactions may be used as a form of 'agonistic buffering' (Deag, 1980; Deag and Crook,

114 1971; Paul et al., 1996). Thus, triadic male-infant-male interaction is a behavior with

115 qualities similar to others that induce the release of OT.

116 Similar to previous studies in chimpanzees and tufted capuchins (Benítez et al., 2018;

117 Crockford et al., 2013), we tested in Barbary macaques whether urinary OT levels were

118 influenced by grooming interactions and - given its functional relevance in Barbary

119 macaques - also by male-infant-male interactions. We predicted that urinary OT levels

120 would be higher after affiliative interactions (i.e., grooming, male-infant-male interactions)

121 compared to a control period without any social interactions. We additionally tested

122 whether the release of OT was partner-specific (i.e., bond partners vs. non-bond partners).

123 If OT release is partner-specific, we predicted that it will be higher after affiliations with

124 bond partners than with non-bond partners. A secondary aim was to test whether OT

125 would generally increase the propensity to cooperate in within-group polyadic agonistic

126 conflicts. To do this under natural conditions, we first determined which affiliative

127 behaviors are positively related to urinary OT levels (as part of the first aim), to use the

128 occurrence of this interaction as a proxy for elevated OT levels in the subject. We

129 consequently predicted, that the probability to cooperate, i.e. accept a solicitation to 
130 support another individual in an agonistic conflict, would be higher after an affiliative

131 interaction.

\section{$132 \quad 2$ Materials and Methods}

\section{$133 \quad 2.1$ Study site and animals}

134 Study subjects belonged to one of three groups of Barbary macaques living together in 14.5

135 ha. of enclosed forest at Affenberg Salem, Germany (de Turckheim and Merz, 1984).

136 Monkeys were provisioned once daily with fruits, vegetables, grains and had ad libitum

137 access to water and monkey chow. Data collection took place from 31 March to 17

138 December 2016, including one non-mating season (31 March to 26 October) and one

139 mating season (27 October to 17 December). The start of the mating season was defined by

140 the first observed ejaculatory copulation. The study group (group C) consisted of 13-14

141 adult males (one male died during the study period), 20 adult females, 2 large subadult

142 males, 8 immature males, 10 immature females and 1 newborn infant male. All members of

143 the group were individually recognized by observers.

\section{$144 \quad 2.2$ Behavioral data collection}

145 Behavioral data were collected from 14 adult males using continuous focal animal sampling

146 (Martin and Bateson, 2007) during individual full-day focal animal follows, in which the

147 occurrence and partners of all social interactions were recorded (Total $=4355$ hours, $311 \pm$

148 SD 40 hours per individual).

\section{$149 \quad 2.3 \quad$ Assessing dyadic bond strength}

150 To assess dyadic bond strength, we calculated the dyadic Composite Sociality Index (CSI;

151 Silk et al., 2010, 2006), with slight modifications as described in Haunhorst et al. (2016).

152 This index ranges from 0 to infinity and has a mean value of 1 , where higher CSI scores

153 indicate a stronger social bond. To calculate the CSI, we chose seven significantly correlated

154 affiliative behavioral variables: duration and count of close proximity $(\leq 1.5$ meters $)$

155 without aggression, duration and count of body contact, duration and count of grooming

156 and count of triadic male-infant-male interactions. Both duration and count of behaviors 
157 were corrected for the total observation time of the dyad. Male Barbary macaques affiliate 158 with females much more frequently than with other males (mean \pm SD behavior seconds

159 per observation hour per sex of dyad: proximity, male-male dyads $=69 \pm 57$; proximity,

160 male-female dyads $=372 \pm 121$; body contact, male-male dyads $=36 \pm 38$; body contact,

161 male-female dyads $=247 \pm 71$; grooming, male-male dyads $=22 \pm 20$; grooming, male-

162 female dyads = $201 \pm 55$ ). If we had included both male-male and male-female dyads into a

163 single CSI scores this would lower the CSI scores for male-male dyads. As a results we

164 would potentially miss-classify some male-male dyads as non-bond when in fact they are

165 bond partners, given that male-male bonds are meaningful and have adaptive significance

166 (see introduction). Therefore, we constructed separate CSI scores for male-male and male-

167 female dyads. The two large subadult males in our study group were included in the

168 calculation of the male-male CSI scores because they supported other adult males in

169 agonistic conflicts. Furthermore, we calculated separate CSI scores for the non-mating and

170 mating seasons as affiliation patterns may change across seasons. Out of the seven

171 affiliative behavior conditions, we only included them in the CSI calculation if their mean

172 frequency of occurrence per dyad in each period was $>2$ to avoid rare behaviors

173 disproportionately affecting the CSI scores. We defined bond partners as those dyads with

174 a CSI score $>1$ (above the group mean).

\section{$1752.4 \quad$ Urine sample collection}

176 Urine samples were collected opportunistically from individuals during focal follows. When

177 monkeys were seen to urinate, the urine was caught with a plastic bag when possible or

178 collected from leaves, branches, rocks or the ground by using a disposable pipette or

179 salivette (Salivette Cortisol, Sarstedt, Nümbrecht, Germany). The use of salivettes to collect

180 urine has recently been validated and successfully applied to urine samples from free-

181 ranging macaques (Danish et al., 2015; Müller et al., 2017). Urine samples contaminated

182 with feces, blood or urine from other individuals were not collected. Urine samples

183 collected by pipette were transferred to $2 \mathrm{ml}$ cryotubes. Both samples stored in cryotubes

184 and salivettes were kept in a thermos flask filled with ice while in the field. At the end of the

185 day, urine was recovered from the salivettes by centrifugation for $5 \mathrm{~min}$ at $1500 \mathrm{rpm}$ using

186 an electric centrifuge and also transferred to $2 \mathrm{ml}$ cryotubes. Samples were split into two 
187 aliquots (100 to $2000 \mu \mathrm{l}$ each). One aliquot was used for analysis of creatinine. In the

188 second aliquot, $0.5 \mathrm{~N}$ phosphoric acid were added to urine at a ratio of 1:10 acid to urine to

189 prevent the breakdown of OT in the sample (Reyes et al., 2014; Ziegler, 2018). All samples

190 were then stored in a freezer at $-20^{\circ} \mathrm{C}$. When data collection was complete, samples were

191 transported in containers with dry ice to the lab and stored once again at $-20^{\circ} \mathrm{C}$.

192 Urine samples were collected from all 14 adult males (7 to 25 years old) of the study group.

193 We presumed a clearance window of 15 to $60 \mathrm{~min}$ for excretion of OT in urine, as done in

194 previous studies investigating urinary OT levels in other non-human primates which show

195 biologically relevant changes in behavior during this window (Benítez et al., 2018;

196 Crockford et al., 2013; Samuni et al., 2017). Studies in humans and marmosets have

197 demonstrated elevated OT levels in urine 30 to 60 after administration of radio-labelled

198 hormone (humans: Amico et al., 1987; marmosets: Seltzer and Ziegler, 2007). Exogenous

199 administration of OT in tufted capuchin monkeys also caused elevated urinary OT levels

200 15-60 min after administration (Benítez et al., 2018). Prior to analysis, urine samples were

201 assigned to different behavioral conditions depending on whether at least one grooming

202 (total time $\geq 60 \mathrm{sec}$ ), triadic male-infant-male interaction or no social interactions occurred

203 in the 45 min clearance window. As we were interested in the role of OT in bonding in a

204 non-sexual context, we only considered samples collected during the non-mating season

205 for analysis. Furthermore, samples were excluded from analysis if any ejaculatory

206 copulations, play or coalitions co-occurred in the clearance window because these

207 behaviors could potentially influence OT levels and confound results. This left us with 76

208 non-social (control) samples (mean $=5.8$, range $=2-11$ per individual) and 63 samples

209 where at least one affiliation occurred (test samples: mean $=4.8$, range $=1-9$ per

210 individual).

\section{$2112.5 \quad$ Extraction and hormone analysis}

212 The extraction and analysis of OT followed a protocol described in detail in Samuni et al.

213 (2017). Briefly, urine samples were thawed and kept cool using an Iso-rack $\left(0^{\circ} \mathrm{C}\right.$;

214 Eppendorf). Then samples were centrifuged for $1 \mathrm{~min}$ at $1500 \mathrm{rpm}$ at $4^{\circ} \mathrm{C}$. Solid-phase

215 extraction cartridges (Chromabond HR-X, 30mg, $1 \mathrm{ml}$, Macherey-Nagel, Dueren, Germany) 
216 were conditioned with $1 \mathrm{ml} \mathrm{MeOH}$ followed by $1 \mathrm{ml}$ distilled HPLC-water. Cartridges were

217 then filled with up to $1 \mathrm{ml}$ dilution buffer (water, $0.1 \%$ TFA) and 20 to $100 \mu \mathrm{l}$ of urine.

218 Diluted urine was allowed to run through the cartridge. Then, the cartridge was washed

219 with $1 \mathrm{ml}$ washing solution (10\% ACN, 1\% TFA) and dried using a vacuum. Hormones were

220 eluted using $1 \mathrm{ml}$ ACN $80 \%$ into clean test tubes. Elutes were evaporated at $50^{\circ} \mathrm{C}$ with

221 pressurized air. Then $300 \mu \mathrm{EtOH} \mathrm{100 \%} \mathrm{was} \mathrm{added} \mathrm{to} \mathrm{each} \mathrm{test} \mathrm{tube} \mathrm{and} \mathrm{shaken} \mathrm{gently.}$

222 Test tubes were allowed to sit for 1 hour at $4^{\circ} \mathrm{C}$ to precipitate proteins before being

223 evaporated again at $50^{\circ} \mathrm{C}$. Samples were then reconstituted with $250 \mu \mathrm{l}$ assay buffer from a

224 commercially available enzyme immunoassay kit (Assay Designs; 901-153A-0001), and

225 vortexed gently for $10 \mathrm{sec}$ by hand. Extracts were then transferred to $1.5 \mathrm{ml}$ labeled

226 eppendorf tubes, and vortexed for $1 \mathrm{~min}$ at 10,000 rpm. Extracts were then kept cool on ice

227 while preparing the assay. The assay was then performed according to instructions

228 provided by the manufacturer.

229 To determine the efficiency of the extraction protocol, we created 5 pools of Barbary

230 macaque urine samples. Before extraction, $75 \mu \mathrm{l}$ of each pooled sample were spiked with

$23175 \mu \mathrm{l}$ of an OT standard $(1500 \mathrm{pg} / \mathrm{ml})$. We used the values from the spiked and unspiked

232 samples to calculate percent recovery for extraction efficiency and assay accuracy

233 following the formula given in Behringer et al. (2012). Mean extraction was 81.0\% (range:

$23492.7 \%-68.7 \%, \mathrm{SD}=10.2, \mathrm{~N}=5$ ). We investigated matrix effects that could potentially

235 interfere with the assay system by testing for parallelism. Out of a pool sample, we took 3

$236 \mathrm{ml}$ of urine and extracted them according to our extraction protocol. Of the resulting $500 \mu \mathrm{l}$

237 of extract, $250 \mu \mathrm{l}$ were taken and serially diluted. Another $1 \mathrm{ml}$ of the urine pool sample

238 was mixed with $100 \mu \mathrm{l}$ of an OT standard solution (10 $000 \mathrm{pg} / \mathrm{ml})$, extracted and serially

239 diluted as described above. Dilutions of the spiked and unspiked pool sample were then

240 brought to assay. Serially diluted pool samples of spiked and unspiked Barbary macaque

241 urine were parallel to the standard as confirmed by visual inspection (Fig. S1).

242 The assay standard curve ranged from 15.62 to $1000 \mathrm{pg} / \mathrm{ml}$ and assay sensitivity at $90 \%$

243 binding was $30 \mathrm{pg} / \mathrm{ml}$. Intra-assay coefficients of variation (CV) of high and low value

244 quality controls were 5.2\% (high) and 31.3\% (low) while respective figures for inter-assay

245 CVs were $11.0 \%$ (high) and 19.7\% (low). 
246 Urinary OT concentrations were corrected for levels of creatinine to account for differences

247 in volume and concentration of excreted urine (Bahr et al., 2000), and are expressed as

$248 \mathrm{pg} / \mathrm{mg}$ creatinine. Because very low concentrations of creatinine may lead to an

249 overestimation of hormone concentration we excluded all samples $(\mathrm{N}=3)$ with $<0.5$

$250 \mathrm{mg} / \mathrm{ml}$ creatinine.

\section{$251 \quad 2.6 \quad$ Statistical analysis}

252 To test whether affiliative interactions influenced urinary OT levels, we fitted two Bayesian 253 multilevel linear regression models (model 1a, b) with a Gaussian response distribution

254 and identity link function. To test whether the probability to give support in an agonistic

255 encounter after being recruited was influenced by a previous grooming interaction, we

256 fitted a Bayesian multilevel linear regression model with a Bernoulli response distribution

257 and logit link function. We included male identity as a random effect in all models. In all

258 models, predictor variables varied within male identity and therefore we included random

259 slopes as well as correlation parameters between random intercepts and random slopes

260 into the models (Barr et al., 2013; Schielzeth and Forstmeier, 2009). We fitted models using

261 the computational framework Stan (https://mc-stan.org), called via R (version 3.5.2; R

262 Core Team, 2018) by using the function brm from the package brms (version 2.9.0;

263 Bürkner, 2017). We ran all models with 5000 iterations over four MCMC chains including

264 an initial 1000 "warm up" iterations for each chain, resulting in a total of 16000 posterior

265 samples (Bürkner, 2017). In all models, we deemed the MCMC results as reliable because

266 there were no divergent transitions during warm up, all Rhat values were equal to 1.00 and

267 visual inspection of a plot of the chains showed that they were able to converge. We used a

268 set of weakly informative priors to improve convergence, guard against overfitting and

269 regularize parameter estimates (Lemoine, 2019; McElreath, 2016): for the intercept and

270 beta coefficients we used a normal distribution with mean 0 and standard deviation 10; for

271 the standard deviation of group level (random) effects and sigma we used a Half-Cauchy

272 distribution with location 0 and scale parameter 1; for the correlation between random

273 slopes we used LKJ Cholesky prior with eta 2. 
274 For all models, we report the estimate as the mean of the posterior distribution and 95\%

275 credible intervals (CI). We calculated the proportion of the posterior samples that fall on

276 the same side of 0 as the mean. This may be interpreted as the probability (Pr) that a given

277 predictor was associated with an outcome, where $\operatorname{Pr}=1$ indicates that the estimate was

278 entirely positive or negative and $\mathrm{Pr}=0.5$ indicates that the estimate is centered around 0

279 and thus the predictor likely had no effect.

\subsubsection{Effect of affiliation on urinary OT levels}

281 To test whether urinary OT levels were generally influenced by affiliative behaviors we

282 fitted two models. As the response we log-transformed urinary OT levels to achieve a more 283 symmetrical distribution. In model 1a, we tested for a general effect of affiliation and 284 included one categorical predictor where OT levels following grooming and triadic male-

285 infant-male interactions were compared to non-social controls. In model 1b, we tested 286 whether OT levels would be influenced differently after affiliation with bond versus non-

287 bond partners. Therefore, we split samples after triadic male-infant-male interactions into 288 bond and non-bond partner categories. As we were only able to collect two urine samples 289 where focal males groomed with a non-bond partner, we decided not to split grooming 290 samples according to partner bond strength.

\section{$291 \quad$ 2.6.2 Effect of grooming on probability to give support}

292 To test whether the probability to give support in an agonistic encounter (between adult 293 and/or subadult individuals) after being recruited was influenced by a previous grooming

294 interaction, we fitted one model. As the response, we included whether our focal male

295 supported another adult individual following a recruit attempt (no/yes). As a test

296 predictor, we included whether our focal animal was in a grooming interaction ( $\geq 60 \mathrm{sec})$

297 with an adult individual within 15-60 min before the recruit behavior (no/yes). This time

298 window was chosen because intranasal administration of OT in rhesus macaques

299 influenced social behaviors up to two hours after inhalation (Chang et al., 2012). Therefore,

300 we chose a comparatively conservative window of 15-60 min for when naturally centrally

301 released OT may still exert behavioral effects. As a control predictor, we included the bond

302 strength of the focal animal to the recruiter (non-bond/bond). 


\section{$303 \quad 3$ Results}

304 We first tested for a general effect of affiliative interactions (grooming and male-infant-

305 male triadic interactions) irrespective of partner bond strength on urinary OT levels.

306 Urinary OT levels were substantially higher after grooming with any partner compared to

307 non-social controls (mean \pm SD OT: non-social: $357 \pm 400$ pg/mg creatinine; grooming: 589

$308 \pm 612 \mathrm{pg} / \mathrm{mg}$ creatinine; average increase of $65 \%$; $\mathrm{Pr}=0.97$; Table 1 a, b; Fig. 1; Fig. 2),

309 while this was not the case for male-infant-male interactions with any partner (mean \pm SD

310 OT: male-infant-male: $455 \pm 438$ pg/mg creatinine; $\operatorname{Pr}=0.77$; Table 1).

311 When we separated male-infant-male interaction samples by bond strength, urinary OT

312 levels after male-infant-male interactions with bond partners were also not substantially

313 different from non-social controls (mean \pm SD OT: non-social: $357 \pm 400 \mathrm{pg} / \mathrm{mg}$ creatinine;

314 male-infant-male bond: $360 \pm 410 \mathrm{pg} / \mathrm{mg}$ creatinine;). In contrast, urinary OT levels were

315 substantially higher after triadic male-infant-male interactions with non-bond partners

316 than non-social controls (mean \pm SD OT: male-infant-male non-bond: $600 \pm 456 \mathrm{pg} / \mathrm{mg}$

317 creatinine; average increase of 68\%; $\mathrm{Pr}=0.96$; Table 1 b; Fig. 1; Fig. 2). 
318 Table 1: Results of models $1 \mathrm{a}$ and $1 \mathrm{~b}$ testing effect of different affiliation conditions on

319 urinary OT levels. In both models, male identity was included as a random effect, $\mathrm{N}=13$

320 males, $\mathrm{N}=139$ samples. $\mathrm{CI}=95 \%$ credible intervals, $\mathrm{Pr}=$ proportion of the posterior

321 samples that fall on the same side of 0 as the mean.

\section{Estimate SD CI lower CI upper Pr}

(a)

Intercept

5.48

$0.12 \quad 5.25$

5.71

1.00

Groom

0.47

$0.25-0.01$

0.99

0.97

Male-infant-male

0.17

$0.23-0.30$

0.60

0.77

(b)

Intercept

Groom

Male-infant-male bond

Male-infant-male non-bond
5.49

0.47

$-0.05$

$\begin{array}{ll}0.28 & -0.59\end{array}$

0.57

$\begin{array}{ll}0.33 & -0.09\end{array}$
1.22

0.96

1.00

0.97

$0.51 \quad 0.58$

1.22 


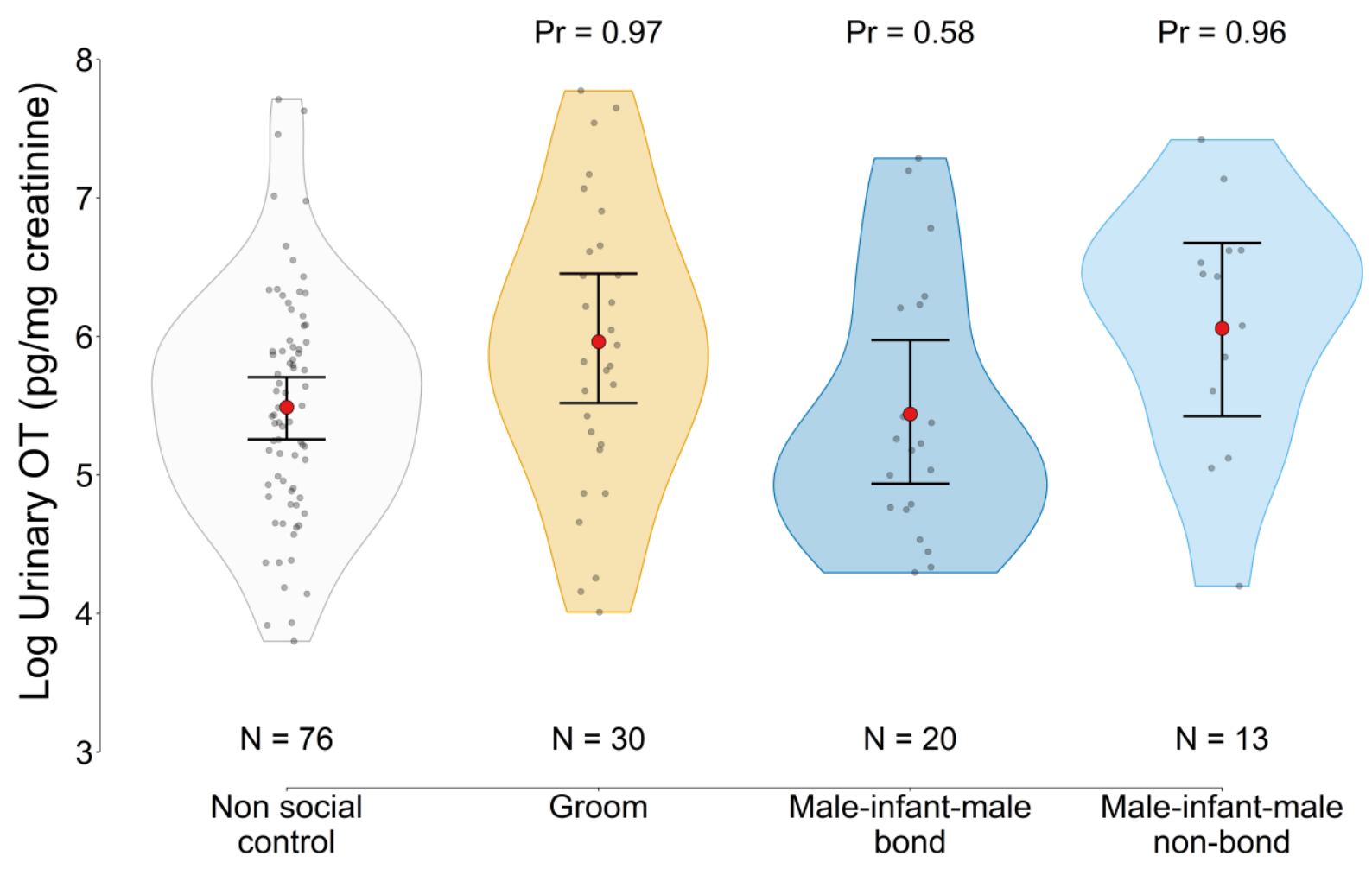

323

324 Fig. 1: Urinary OT levels per behavioral condition. Violin plots show the density of observed

325 data points. Solid red dots show fitted values from model 1b: mean of posterior

326 distribution and 95\% credible intervals. $\operatorname{Pr}=$ proportion of the posterior samples that fall

327 on the same side of 0 as the mean. $\mathrm{N}=$ number of samples per condition. 


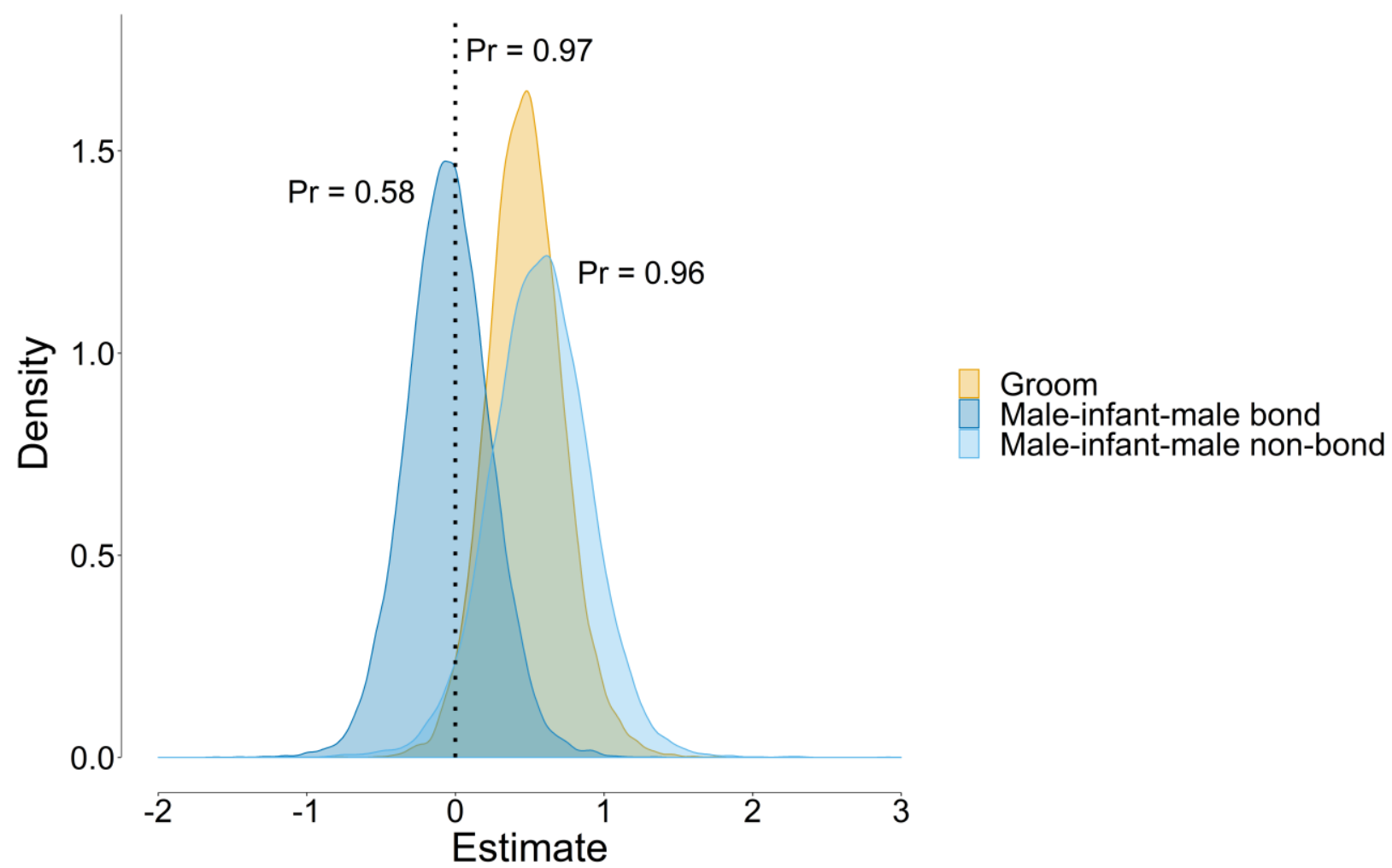

330 Fig. 2: Posterior probability distribution of the difference in urinary OT levels after

331 different affiliative behaviors compared to non-social controls. $\mathrm{Pr}=$ proportion of the

332 posterior samples that fall on the same side of 0 as the mean.

\subsection{Effect of grooming on probability to give support}

334 We recorded a total 205 attempts of adult individuals to recruit the focal animal for an

335 agonistic conflict. In 64 (31\%) cases the focal animal supported the recruiter and in 67

$336(33 \%)$ cases the focal animal was in a grooming interaction with any adult group member

33715 to 60 minutes prior to the recruitment attempt (these samples are not mutually

338 exclusive). In only 7 (3\%) cases were the previous grooming partner also the recruiter.

339 Grooming interactions did not substantially influence the probability to support a recruiter

340 in an agonistic encounter within 15 to 60 minutes after the grooming interaction (N

341 support given when groomed before $=16$, N support given when not groomed before $=48$;

342 Pr = 0.86; Table 2). 
343 Table 2: Model 2 results testing the effect of grooming on the probability to give support in

344 an agonistic conflict after being recruited. Bond strength with the recruiter was included as

345 a control variable. $\mathrm{N}=14$ males, $\mathrm{N}=205$ observations. $\mathrm{CI}=95 \%$ credible intervals, $\mathrm{Pr}=$

346 proportion of the posterior samples that fall on the same side of 0 as the mean.

$\begin{array}{llllll} & \text { Estimate } & \text { SD } & \text { CI lower } & \text { CI upper } & \text { Pr } \\ \text { Intercept } & -1.05 & 0.35 & -1.80 & -0.41 & 1.00 \\ \text { Groom before? (yes) } & -0.45 & 0.43 & -1.32 & 0.37 & 0.86 \\ \text { Recruiter bond (bond) } & 0.81 & 0.46 & -0.03 & 1.82 & 0.97\end{array}$

\section{Discussion}

349 Overall we found a high probability that urinary OT levels are elevated following grooming

350 interactions in adult male Barbary macaques. This is generally in line with previous studies

351 showing a positive relationship between OT and grooming (primates: Benítez et al., 2018;

352 Crockford et al., 2013; Snowdon et al., 2010; vampire bats, Desmodus rotundus: Carter and

353 Wilkinson, 2015), as well as other socio-positive interactions more generally (primates:

354 Preis et al., 2018; Benítez et al., 2018; Snowdon et al., 2010; Wittig et al., 2014; vampire

355 bats: Carter and Wilkinson, 2015; dogs: Romero et al., 2014). Given the low number of

356 grooming between non-bonded partners in our study, we could not test partner specific

357 effects of grooming. In chimpanzees, oxytocin release was partner-specific in one

358 population, with elevations after grooming with a bond, yet not with a non-bond partner

359 (Crockford et al., 2013); in another population OT levels were generally increased after

360 affiliation (including grooming) irrespective of partner bond strength (Preis et al., 2018).

361 Relationship quality was tested differently in these two studies, with relationship quality

362 being either categorized dichotomously into bond and non-bond partners (Crockford et al.,

363 2013), or being tested on a continuous scale (Preis et al., 2018). Our cut-off relationship

364 strength value for classification as a bond partner was much lower than the one used for 
365 chimpanzees. We do not know how nonhuman primates classify each other into

366 biologically meaningful bond and non-bond categories, e.g. an inner clique of 2-3 bonded

367 partners (Hill et al., 2008; Zhou et al., 2005), and if this mental classification mediates OT

368 release. In principle there is good evidence that classification into bond partners affects

369 physiological responses to social interactions. The social buffering phenomenon shows that

370 the presence or interaction with closely bonded partners during stressful events mitigates

371 the release of glucocorticoids (Hennessy et al., 2009; Kikusui et al., 2006; Wittig et al., 2016;

372 Young et al., 2014a) with OT release mediating social buffering of the stress response

373 (Crockford et al., 2017; Hennessy et al., 2009; Kikusui et al., 2006; Smith and Wang, 2014).

374 Unexpectedly, urinary OT levels were elevated after triadic male-infant-male interactions

375 with non-bond partners, but not after interactions with bond partners. This finding

376 contradicts the idea that it is affiliation with bond rather than non-bond partners that

377 triggers the release of OT (Crockford et al., 2013; Finkenwirth et al., 2015). This could

378 indicate that male-infant-male interactions serve to promote the formation of social bonds

379 with not yet bonded partners, while physiologically not impacting interactions between

380 established partners. An untested, yet possible alternative given the correlational nature of

381 our study is the reversed cause-effect directionality: instead of a male-infant-male

382 interaction triggering the release of OT, OT may increase the probability of a male-infant-

383 male interaction to occur. In this scenario, male-infant-male interactions do not function in

384 bond formation, but for other reasons, for example as a form of "agonistic buffering" (Deag,

385 1980; Deag and Crook, 1971; Paul et al., 1996). In support of this idea, rates of male-infant-

386 male interactions increase during tense feeding situations while other types of affiliation

387 (such as grooming) decrease (Paul et al., 1996). Social relationships between adult males

388 are generally tense and affiliation between them often takes place in the presence of infants

389 (Deag, 1980; Preuschoft and Paul, 2000). Due to the anxiolytic effect of OT (Neumann and

390 Landgraf, 2012), elevated levels of OT may facilitate male-infant-male interactions via

391 increasing the motivation to approach and at the same time reducing avoidance behaviors

392 toward other males (Kemp and Guastella, 2011). Reduced anxiety may be particularly

393 useful for interacting with non-bond males with whom the relationship is presumably

394 more tense and unpredictable than with bond partners (Young et al., 2014b). Such an 
explanation would be consistent with our finding that OT levels were elevated after maleinfant-male interactions with non-bond partners. While intriguing, we need to stress our small sample size of male-infant-male interactions with non-bond partners as well as previous work on this and other species pointing to the bond strengthening and cooperation-enhancing function of male-infant-male interactions (Berghänel et al., 2011;

400 Kalbitz et al., 2017). Thus, additional studies able to disentangle OT levels directly before 401 and after affiliative interactions are clearly needed for a more conclusive picture.

402 Prior grooming did not increase the probability of supporting a group member in a conflict.

403 This test builds on the assumption that after engaging in grooming OT levels will be 404 elevated, potentially influencing behavior and more specifically cooperative tendency. If 405 true, this finding suggests that OT did not increase a male's general tendency to cooperate 406 in a conflict, which is maybe not surprising given the wealth of studies indicating that OT's 407 prosocial effects depend on situational context and interaction partner (Bartz et al., 2011). 408 In an economic game, human cooperation was enhanced by intranasal OT administration 409 only if participants had prior contact yet not with strangers (Declerck et al., 2010), and 410 similarly, female house mice (Mus musculus domesticus) receiving OT actually decreased 411 the propensity to cooperate in communal breeding with strangers (Harrison et al., 2017).

412 In another study in humans intranasal OT increased trust but not if the partner was

413 portrayed as untrustworthy (Mikolajczak et al., 2010). Thus, the social information on a

414 partner is an important component for OT-induced cooperation and depending on this

415 information OT may reduce the propensity to cooperate.

416 Our results do not exclude the possibility that OT promotes direct or partner-specific 417 cooperation. From a behavioral perspective, individuals are more likely to support others 418 with whom they have groomed in the recent past (long-tailed macaques, Macaca 419 fascicularis: Hemelrijk, 1994; chacma baboons, Papio ursinus: Cheney et al., 2010). In our 420 study there were only a few cases of the former grooming partner asking for help within 421 the next hour, therefore we could not explicitly test this scenario. Contingent cooperation 422 appears to be rare in animals, and more commonly support is given less strictly on a 423 contingent basis but instead to bonded partners who form long term alliances (Cheney, 424 2011). OT would then mediate cooperation with specific partners through its role in 
425 promoting the formation of social bonds. While we did not have an explicit aim to test the

426 effect of partner bond strength on the probability to cooperate, this variable was included

427 in our model as a control predictor, and it did substantially increase the probability of

428 giving support. Preferentially giving support to bonded partners has also previously been

429 shown in this (Young et al., 2014b) and other species (Schülke et al., 2010; Smith et al.,

430 2011; Watts, 2002).

431 Overall, our study adds to the body of research indicating that OT is involved in the

432 regulation of adult social bonds, including in non-reproductive contexts. Questions still

433 remain under which contexts OT release is partner specific. It has been suggested that in

434 smaller social groups, all group members are bonded to a sufficient degree to elicit OT

435 release after affiliative interactions (Benítez et al., 2018), whereas in larger groups variance

436 in affiliation rates may be large enough that OT release may only occur after affiliations

437 with more closely bonded partners. Such an explanation would be consistent with

438 observed differences in partner specificity of OT release between chimpanzee populations

439 (Crockford et al., 2013; Preis et al., 2018). Finally, the lack of an effect of male-infant-male

440 interactions on OT levels in male Barbary macaques, at least with bond partners and

441 perhaps overall, remains puzzling. Particularly so given that behaviors other than

442 grooming that potentially promote social bonding also increased urinary OT levels in other

443 species (Benítez et al., 2018; Romero et al., 2014; Wittig et al., 2014). One difference

444 between grooming and male-infant-male interactions is that male-infant-male interactions

445 are more ritualistic in nature. Perhaps the bonding effect of ritualized behavior may be

446 under the control of other neuropeptides, such as endorphins, as has been shown for

447 synchronous dancing in humans (Tarr et al., 2015, 2016).

\section{Acknowledgements}

449 We thank Ellen Merz and Roland Hilgartner for permission to conduct the study at 450 Affenberg Salem. We thank Lauren Cassidy, Tatjana Kaufmann and Lilah Sciaky for help

451 with urine sample and behavioral data collection. We are grateful to Vera Schmeling for

452 general assistance in the lab. This research was funded by the Deutsche

453 Forschungsgemeinschaft (DFG, German Research Foundation) - Project number

454254142454 / GRK 2070. 


\section{References}

456 Amico, J.A., Ulbrecht, J.S., Robinson, A.G., 1987. Clearance studies of oxytocin in humans

457 using radioimmunoassay measurements of the hormone in plasma and urine. J. Clin.

458 Endocrinol. Metab. 64, 340-345. https://doi.org/10.1210/jcem-64-2-340

459 Archie, E.A., Tung, J., Clark, M., Altmann, J., Alberts, S.C., 2014. Social affiliation matters: Both

460 same-sex and opposite-sex relationships predict survival in wild female baboons. Proc. R.

461 Soc. B 281, 20141261. https://doi.org/10.1098/rspb.2014.1261

462 Arueti, M., Perach-Barzilay, N., Tsoory, M.M., Berger, B., Getter, N., Shamay-Tsoory, S.G.,

463 2013. When two become one: The role of oxytocin in interpersonal coordination and

464 cooperation. J. Cogn. Neurosci. 25, 1418-1427. https://doi.org/10.1162/jocn_a_00400

465 Bahr, N.I., Palme, R., Möhle, U., Hodges, J.K., Heistermann, M., 2000. Comparative aspects of

466 the metabolism and excretion of cortisol in three individual nonhuman primates. Gen.

467 Comp. Endocrinol. 117, 427-438. https://doi.org/10.1006/gcen.1999.7431

468 Barr, D.J., Levy, R., Scheepers, C., Tily, H.J., 2013. Random effects structure for confirmatory 469 hypothesis testing: Keep it maximal. J. Mem. Lang. 68, 255-278.

470 https://doi.org/10.1016/j.jml.2012.11.001

471 Bartz, J.A., Zaki, J., Bolger, N., Ochsner, K.N., 2011. Social effects of oxytocin in humans:

472 Context and person matter. Trends Cogn. Sci. 15, 301-309.

473 https://doi.org/10.1016/j.tics.2011.05.002

474 Behringer, V., Hohmann, G., Stevens, J.M.G., Weltring, A., Deschner, T., 2012. Adrenarche in

475 bonobos (Pan paniscus): Evidence from ontogenetic changes in urinary

476 dehydroepiandrosterone-sulfate levels. J. Endocrinol. 214, 55-65.

477 https://doi.org/10.1530/JOE-12-0103

478 Benítez, M.E., Sosnowski, M.J., Tomeo, O.B., Brosnan, S.F., 2018. Urinary oxytocin in

479 capuchin monkeys: Validation and the influence of social behavior. Am. J. Primatol. 80,

480 e22877. https://doi.org/10.1002/ajp.22877 
481 Berghänel, A., Ostner, J., Schröder, U., Schülke, 0., 2011. Social bonds predict future

482 cooperation in male Barbary macaques, Macaca sylvanus. Anim. Behav. 81, 1109-1116.

483 https://doi.org/10.1016/j.anbehav.2011.02.009

484 Bürkner, P.-C., 2017. Brms: An R package for Bayesian multilevel models using Stan. J. Stat.

485 Softw. 80, 1-28. https://doi.org/10.18637/jss.v080.i01

486 Cameron, E.Z., Setsaas, T.H., Linklater, W.L., 2009. Social bonds between unrelated females

487 increase reproductive success in feral horses. Proc. Natl. Acad. Sci. 106, 13850-13853.

488 https://doi.org/10.1073/pnas.0900639106

489 Carter, G.G., Wilkinson, G.S., 2015. Intranasal oxytocin increases social grooming and food

490 sharing in the common vampire bat Desmodus rotundus. Horm. Behav. 75, 150-153.

491 https://doi.org/10.1016/j.yhbeh.2015.10.006

492 Chang, S.W.C., Barter, J.W., Ebitz, R.B., Watson, K.K., Platt, M.L., 2012. Inhaled oxytocin

493 amplifies both vicarious reinforcement and self reinforcement in rhesus macaques (. Proc.

494 Natl. Acad. Sci. 109, 959-964. https://doi.org/10.1073/pnas.1114621109

495 Cheney, D.L., 2011. Extent and limits of cooperation in animals. Proc. Natl. Acad. Sci. 108,

496 10902-10909. https://doi.org/10.1073/pnas.1100291108

497 Cheney, D.L., Moscovice, L.R., Heesen, M., Mundry, R., Seyfarth, R.M., 2010. Contingent

498 cooperation between wild female baboons. Proc. Natl. Acad. Sci. 107, 9562-9566.

499 https://doi.org/10.1073/pnas.1001862107

500 Cheney, D.L., Seyfarth, R.M., 2009. Stress and coping mechanisms in female primates, in:

501 Advances in the Study of Behavior. Academic Press, pp. 1-44.

502 https://doi.org/10.1016/S0065-3454(09)39001-4

503 Crockford, C., Deschner, T., Wittig, R.M., 2017. The role of oxytocin in social buffering: What

504 do primate studies add?, in: Hurlemann, R., Grinevich, V. (Eds.), Behavioral Pharmacology

505 of Neuropeptides: Oxytocin. Springer, Cham, pp. 155-173.

506 https://doi.org/10.1007/7854_2017_12 
507 Crockford, C., Wittig, R.M., Langergraber, K., Ziegler, T.E., Zuberbühler, K., Deschner, T.,

508 2013. Urinary oxytocin and social bonding in related and unrelated wild chimpanzees.

509 Proc. R. Soc. B 280, 20122765. https://doi.org/10.1098/rspb.2012.2765

510 Danish, L.M., Heistermann, M., Agil, M., Engelhardt, A., 2015. Validation of a novel collection

511 device for non-invasive urine sampling from free-ranging animals. PLoS ONE 10, e0142051.

512 https://doi.org/10.1371/journal.pone.0142051

513 Deag, J.M., 1980. Interactions between males and unweaned Barbary macaques: Testing the

514 agonistic buffering hypothesis. Behaviour 75, 54-80.

515 https://doi.org/10.1163/156853980X00564

516 Deag, J.M., Crook, J.H., 1971. Social behaviour and "agonistic buffering” in the wild Barbary

517 macaque Macaca sylvana L. Folia Primatol. 15, 183-200.

518 https://doi.org/10.1159/000155378

519 Declerck, C.H., Boone, C., Kiyonari, T., 2010. Oxytocin and cooperation under conditions of

520 uncertainty: The modulating role of incentives and social information. Horm. Behav. 57,

521 368-374. https://doi.org/10.1016/j.yhbeh.2010.01.006

522 De Dreu, C.K.W., Greer, L.L., Handgraaf, M.J.J., Shalvi, S., Kleef, G.A.V., Baas, M., Velden, F.S.T.,

523 Dijk, E.V., Feith, S.W.W., 2010. The neuropeptide oxytocin regulates parochial altruism in

524 intergroup conflict among humans. Science 328, 1408-1411.

525 https://doi.org/10.1126/science.1189047

526 de Turckheim, G., Merz, E., 1984. Breeding Barbary macaques in outdoor open enclosures,

527 in: Fa, J.E. (Ed.), The Barbary Macaque: A Case Study in Conservation. Springer US, Boston,

528 MA, pp. 241-261. https://doi.org/10.1007/978-1-4613-2785-1_10

529 Dölen, G., Darvishzadeh, A., Huang, K.W., Malenka, R.C., 2013. Social reward requires

530 coordinated activity of nucleus accumbens oxytocin and serotonin. Nature 501, 179-184.

531 https://doi.org/10.1038/nature12518 
532 Finkenwirth, C., Martins, E., Deschner, T., Burkart, J.M., 2016. Oxytocin is associated with

533 infant-care behavior and motivation in cooperatively breeding marmoset monkeys. Horm.

534 Behav. 80, 10-18. https://doi.org/10.1016/j.yhbeh.2016.01.008

535 Finkenwirth, C., van Schaik, C., Ziegler, T.E., Burkart, J.M., 2015. Strongly bonded family

536 members in common marmosets show synchronized fluctuations in oxytocin. Physiol.

537 Behav. 151, 246-251. https://doi.org/10.1016/j.physbeh.2015.07.034

538 French, J.A., Cavanaugh, J., Mustoe, A.C., Carp, S.B., Womack, S.L., 2018. Social monogamy in 539 nonhuman primates: Phylogeny, phenotype, and physiology. J. Sex Res. 55, 410-434.

540 https://doi.org/10.1080/00224499.2017.1339774

541 Frère, C.H., Krützen, M., Mann, J., Connor, R.C., Bejder, L., Sherwin, W.B., 2010. Social and

542 genetic interactions drive fitness variation in a free-living dolphin population. Proc. Natl.

543 Acad. Sci. 107, 19949-19954. https://doi.org/10.1073/pnas.1007997107

544 Giles, L.C., Glonek, G.F.V., Luszcz, M.A., Andrews, G.R., 2005. Effect of social networks on 10

545 year survival in very old Australians: The Australian longitudinal study of aging. J.

546 Epidemiol. Community Health 59, 574-579. https://doi.org/10.1136/jech.2004.025429

547 Harrison, N., C. Lopes, P., König, B., 2017. Oxytocin administration during early pair

548 formation delays communal nursing in female house mice. Anim. Behav. 123, 61-68.

549 https://doi.org/10.1016/j.anbehav.2016.10.030

550 Haunhorst, C.B., Heesen, M., Ostner, J., Schülke, O., 2017. Social bonds with males lower the 551 costs of competition for wild female Assamese macaques. Anim. Behav. 125, 51-60.

552 https://doi.org/10.1016/j.anbehav.2017.01.008

553 Haunhorst, C.B., Schülke, O., Ostner, J., 2016. Opposite-sex social bonding in wild Assamese

554 macaques. Am. J. Primatol. 78, 872-882. https://doi.org/10.1002/ajp.22554

555 Hemelrijk, C.K., 1994. Support for being groomed in long-tailed macaques, Macaca

556 fascicularis. Anim. Behav. 48, 479-481. https://doi.org/10.1006/anbe.1994.1264 
557 Henkel, S., Heistermann, M., Fischer, J., 2010. Infants as costly social tools in male Barbary

558 macaque networks. Anim. Behav. 79, 1199-1204.

559 https://doi.org/10.1016/j.anbehav.2010.02.005

560 Hennessy, M.B., Kaiser, S., Sachser, N., 2009. Social buffering of the stress response:

561 Diversity, mechanisms, and functions. Front. Neuroendocrinol. 30, 470-482.

562 https://doi.org/10.1016/j.yfrne.2009.06.001

563 Hill, R.A., Bentley, R.A., Dunbar, R.I.M., 2008. Network scaling reveals consistent fractal

564 pattern in hierarchical mammalian societies. Biol. Lett. 4, 748-751.

565 https://doi.org/10.1098/rsbl.2008.0393

566 Kalbitz, J., Ostner, J., Schülke, O., 2016. Strong, equitable and long-term social bonds in the

567 dispersing sex in Assamese macaques. Anim. Behav. 113, 13-22.

568 https://doi.org/10.1016/j.anbehav.2015.11.005

569 Kalbitz, J., Schülke, O., Ostner, J., 2017. Triadic male-infant-male interaction serves in bond 570 maintenance in male Assamese macaques. PLoS ONE 12, e0183981.

571 https://doi.org/10.1371/journal.pone.0183981

572 Kemp, A.H., Guastella, A.J., 2011. The role of oxytocin in human affect: A novel hypothesis.

573 Curr. Dir. Psychol. Sci. 20, 222-231. https://doi.org/10.1177/0963721411417547

574 Kikusui, T., Winslow, J.T., Mori, Y., 2006. Social buffering: Relief from stress and anxiety.

575 Philos. Trans. R. Soc. B 361, 2215-2228. https://doi.org/10.1098/rstb.2006.1941

576 Kulik, L., Muniz, L., Mundry, R., Widdig, A., 2012. Patterns of interventions and the effect of

577 coalitions and sociality on male fitness. Mol. Ecol. 21, 699-714.

578 https://doi.org/10.1111/j.1365-294X.2011.05250.x

579 Küster, J., Paul, A., 1992. Influence of male competition and female mate choice on male

580 mating success in Barbary macaques (Macaca sylvanus). Behaviour 120, 192-216.

581 https://doi.org/10.1163/156853992X00606 
582 Lemoine, N.P., 2019. Moving beyond noninformative priors: Why and how to choose

583 weakly informative priors in Bayesian analyses. Oikos 128, 912-928.

584 https://doi.org/10.1111/oik.05985

585 Love, T.M., 2014. Oxytocin, motivation and the role of dopamine. Pharmacol. Biochem.

586 Behav., The role of oxytocin in positive affect and drug-related reward 119, 49-60.

587 https://doi.org/10.1016/j.pbb.2013.06.011

588 Martin, P., Bateson, P., 2007. Measuring behaviour: An introductory guide, 3rd ed.

589 Cambridge University Press, Cambridge.

590 Massen, J.J.M., Sterck, E.H.M., 2013. Stability and durability of intra- and intersex social

591 bonds of captive rhesus macaques (Macaca mulatta). Int. J. Primatol. 34, 770-791.

592 https://doi.org/10.1007/s10764-013-9695-7

593 McElreath, R., 2016. Statistical Rethinking : A Bayesian Course with Examples in R and Stan.

594 Chapman and Hall/CRC. https://doi.org/10.1201/9781315372495

595 Mikolajczak, M., Gross, J.J., Lane, A., Corneille, O., de Timary, P., Luminet, O., 2010. Oxytocin

596 makes people trusting, not gullible. Psychol. Sci. 21, 1072-1074.

597 https://doi.org/10.1177/0956797610377343

598 Müller, N., Heistermann, M., Strube, C., Schülke, O., Ostner, J., 2017. Age, but not

599 anthelmintic treatment, is associated with urinary neopterin levels in semi-free ranging

600 Barbary macaques. Sci. Rep. 7, 41973. https://doi.org/10.1038/srep41973

601 Neumann, I.D., Landgraf, R., 2012. Balance of brain oxytocin and vasopressin: Implications

602 for anxiety, depression, and social behaviors. Trends in Neurosciences 35, 649-659.

603 https://doi.org/10.1016/j.tins.2012.08.004

604 Numan, M., Young, L.J., 2016. Neural mechanisms of mother-infant bonding and pair

605 bonding: Similarities, differences, and broader implications. Horm. Behav., Parental Care

606 77, 98-112. https://doi.org/10.1016/j.yhbeh.2015.05.015

607 Ostner, J., Schülke, O., 2018. Linking sociality to fitness in primates: A call for mechanisms,

608 in: Naguib, M., Barrett, L., Healy, S.D., Podos, J., Simmons, L.W., Zuk, M. (Eds.), Advances in 
609 the Study of Behavior. Academic Press, pp. 127-175.

610 https://doi.org/10.1016/bs.asb.2017.12.001

611 Paul, A., Kuester, J., Arnemann, J., 1996. The sociobiology of male-infant interactions in

612 Barbary macaques, Macaca sylvanus. Anim. Behav. 51, 155-170.

613 https://doi.org/10.1006/anbe.1996.0013

614 Preis, A., Samuni, L., Mielke, A., Deschner, T., Crockford, C., Wittig, R.M., 2018. Urinary

615 oxytocin levels in relation to post-conflict affiliations in wild male chimpanzees (Pan

616 troglodytes verus). Horm. Behav. 105, 28-40. https://doi.org/10.1016/j.yhbeh.2018.07.009

617 Preuschoft, S., Paul, A., 2000. Dominance, egalitarianism, and stalemate: An experimental

618 approach to male-male competition in Barbary macaques, in: Primate Males: Causes and

619 Consequences of Variation in Group Composition. pp. 205-216.

620 R Core Team, 2018. R: A language and environment for statistical computing. R Foundation

621 for Statistical Computing, Vienna, Austria.

622 Reyes, T.L., Galinsky, A.M., Hoffmann, J.N., You, H.M., Ziegler, T.E., McClintock, M.K., 2014.

623 Social peptides: Measuring urinary oxytocin and vasopressin in a home field study of older

624 adults at risk for dehydration. J. Gerontol. B Psychol. Sci. Soc. Sci. 69, S229-S237.

625 https://doi.org/10.1093/geronb/gbu104

626 Romero, T., Nagasawa, M., Mogi, K., Hasegawa, T., Kikusui, T., 2014. Oxytocin promotes

627 social bonding in dogs. Proc. Natl. Acad. Sci. 111, 9085-9090.

628 https://doi.org/10.1073/pnas.1322868111

629 Ross, H.E., Young, L.J., 2009. Oxytocin and the neural mechanisms regulating social

630 cognition and affiliative behavior. Front. Neuroendocrinol., Hormones \& Social Behavior 30,

631 534-547. https://doi.org/10.1016/j.yfrne.2009.05.004

632 Samuni, L., Preis, A., Deschner, T., Crockford, C., Wittig, R.M., 2018. Reward of labor

633 coordination and hunting success in wild chimpanzees. Commun. Biol. 1, 138.

634 https://doi.org/10.1038/s42003-018-0142-3 
635 Samuni, L., Preis, A., Mundry, R., Deschner, T., Crockford, C., Wittig, R.M., 2017. Oxytocin

636 reactivity during intergroup conflict in wild chimpanzees. Proc. Natl. Acad. Sci. 114, 268-

637 273. https://doi.org/10.1073/pnas.1616812114

638 Schielzeth, H., Forstmeier, W., 2009. Conclusions beyond support: Overconfident estimates

639 in mixed models. Behav. Ecol. 20, 416-420. https://doi.org/10.1093/beheco/arn145

640 Schino, G., Aureli, F., 2009. Reciprocal altruism in primates: Partner choice, cognition, and

641 emotions, in: Advances in the Study of Behavior. Academic Press, pp. 45-69.

642 https://doi.org/10.1016/S0065-3454(09)39002-6

643 Schülke, O., Bhagavatula, J., Vigilant, L., Ostner, J., 2010. Social bonds enhance reproductive

644 success in male macaques. Curr. Biol. 20, 2207-2210.

645 https://doi.org/10.1016/j.cub.2010.10.058

646 Seltzer, L.J., Ziegler, T.E., 2007. Non-invasive measurement of small peptides in the common

647 marmoset (Callithrix jacchus): A radiolabeled clearance study and endogenous excretion

648 under varying social conditions. Horm. Behav. 51, 436-442.

649 https://doi.org/10.1016/j.yhbeh.2006.12.012

650 Silk, J.B., Alberts, S.C., Altmann, J., 2006. Social relationships among adult female baboons

651 (Papio cynocephalus) II. Variation in the quality and stability of social bonds. Behav. Ecol.

652 Sociobiol. 61, 197-204. https://doi.org/10.1007/s00265-006-0250-9

653 Silk, J.B., Beehner, J.C., Bergman, T.J., Crockford, C., Engh, A.L., Moscovice, L.R., Wittig, R.M.,

654 Seyfarth, R.M., Cheney, D.L., 2010. Strong and consistent social bonds enhance the longevity

655 of female baboons. Curr. Biol. 20, 1359-1361. https://doi.org/10.1016/j.cub.2010.05.067

656 Skuse, D.H., Gallagher, L., 2009. Dopaminergic-neuropeptide interactions in the social brain.

657 Trends Cogn. Sci. 13, 27-35. https://doi.org/10.1016/j.tics.2008.09.007

658 Small, M.F., 1990. Promiscuity in Barbary macaques (Macaca sylvanus). Am. J. Primatol. 20, 659 267-282. https://doi.org/10.1002/ajp.1350200403 
660 Smith, A.S., Wang, Z., 2014. Hypothalamic oxytocin mediates social buffering of the stress

661 response. Biol. Psychiatry, Neurobiological Moderators of Stress Response 76, 281-288.

662 https://doi.org/10.1016/j.biopsych.2013.09.017

663 Smith, J.E., Powning, K.S., Dawes, S.E., Estrada, J.R., Hopper, A.L., Piotrowski, S.L., Holekamp,

664 K.E., 2011. Greetings promote cooperation and reinforce social bonds among spotted

665 hyaenas. Anim. Behav. 81, 401-415. https://doi.org/10.1016/j.anbehav.2010.11.007

666 Snowdon, C.T., Pieper, B.A., Boe, C.Y., Cronin, K.A., Kurian, A.V., Ziegler, T.E., 2010. Variation

667 in oxytocin is related to variation in affiliative behavior in monogamous, pairbonded

668 tamarins. Horm. Behav. 58, 614-618. https://doi.org/10.1016/j.yhbeh.2010.06.014

669 Strauss, E.D., Holekamp, K.E., 2019. Social alliances improve rank and fitness in convention-

670 based societies. Proc. Natl. Acad. Sci. 201810384.

671 https://doi.org/10.1073/pnas.1810384116

672 Tarr, B., Launay, J., Cohen, Dunbar, R.I.M., 2015. Synchrony and exertion during dance

673 independently raise pain threshold and encourage social bonding. Biol. Lett. 11, 20150767.

674 https://doi.org/10.1098/rsbl.2015.0767

675 Tarr, B., Launay, J., Dunbar, R.I.M., 2016. Silent disco: Dancing in synchrony leads to

676 elevated pain thresholds and social closeness. Evol. Hum. Behav. 37, 343-349.

677 https://doi.org/10.1016/j.evolhumbehav.2016.02.004

678 Ten Velden, F.S., Daughters, K., De Dreu, C.K.W., 2017. Oxytocin promotes intuitive rather

679 than deliberated cooperation with the in-group. Horm. Behav. 92, 164-171.

680 https://doi.org/10.1016/j.yhbeh.2016.06.005

681 Watts, D.P., 2002. Reciprocity and interchange in the social relationships of wild male 682 chimpanzees. Behaviour 139, 343-370.

683 Weidt, A., Hofmann, S.E., König, B., 2008. Not only mate choice matters: Fitness

684 consequences of social partner choice in female house mice. Anim. Behav. 75, 801-808.

685 https://doi.org/10.1016/j.anbehav.2007.06.017 
686 Wittig, R.M., Crockford, C., Deschner, T., Langergraber, K.E., Ziegler, T.E., Zuberbühler, K., 687 2014. Food sharing is linked to urinary oxytocin levels and bonding in related and 688 unrelated wild chimpanzees. Proc. R. Soc. B 281, 20133096.

689 https://doi.org/10.1098/rspb.2013.3096

690 Wittig, R.M., Crockford, C., Weltring, A., Langergraber, K.E., Deschner, T., Zuberbühler, K., 691 2016. Social support reduces stress hormone levels in wild chimpanzees across stressful 692 events and everyday affiliations. Nat. Commun. 7, 13361.

693 https://doi.org/10.1038/ncomms13361

694 Young, C., Hähndel, S., Majolo, B., Schülke, O., Ostner, J., 2013. Male coalitions and female 695 behaviour affect male mating success independent of dominance rank and female receptive 696 synchrony in wild Barbary macaques. Behav. Ecol. Sociobiol. 67, 1665-1677.

697 https://doi.org/10.1007/s00265-013-1577-7

698 Young, C., Majolo, B., Heistermann, M., Schülke, O., Ostner, J., 2014a. Responses to social and 699 environmental stress are attenuated by strong male bonds in wild macaques. Proc. Natl. 700 Acad. Sci. 111, 18195-18200. https://doi.org/10.1073/pnas.1411450111

701 Young, C., Majolo, B., Schülke, O., Ostner, J., 2014b. Male social bonds and rank predict

702 supporter selection in cooperative aggression in wild Barbary macaques. Anim. Behav. 95, 703 23-32. https://doi.org/10.1016/j.anbehav.2014.06.007

704 Young, C., Schülke, O., Ostner, J., 2014c. How males form coalitions against group rivals and 705 the Pandit/van Schaik coalition model. Behaviour 151, 907-934.

706 https://doi.org/10.1163/1568539X-00003166

707 Zhou, W.-X., Sornette, D., Hill, R., Dunbar, R.I.M., 2005. Discrete hierarchical organization of 708 social group sizes. Proc. R. Soc. B 272, 439-444. https://doi.org/10.1098/rspb.2004.2970

709 Ziegler, T.E., 2018. Measuring peripheral oxytocin and vasopressin in nonhuman primates.

710 Am. J. Primatol. 0, e22871. https://doi.org/10.1002/ajp.22871 
711 Ziegler, T.E., Crockford, C., 2017. Neuroendocrine control in social relationships in non-

712 human primates: Field based evidence. Horm. Behav., Field endocrinology of nonhuman

713 primates and humans 91,107-121. https://doi.org/10.1016/j.yhbeh.2017.03.004 